\title{
Interrelation of structural and kinematic characteristics during free-surface gravity flows of granular materials
}

\author{
Viktor N. Dolgunin, Oleg O Ivanov, Andrey N. Kudi, Maxim A. Tuev
}

\begin{abstract}
The interrelationship of flow parameters during free surface gravity flow of cohesionless nonelastic particles is investigated on the basis of a granular medium state equation. The equation establishes the relationship between dilatation, normal pressure and granular temperature. The granular temperature is defined as the sum of three elementary types of kinetic energy of particles in the course of its mutual displacements: relative shear movement, chaotic fluctuation and transversal mass transfer. The investigation was carried out by means of the earlier developed experimental and analytical method presupposing the distribution analysis of particles, falling from the discharge threshold of a rough chute. The profiles of velocity and fraction of the void volume have been determined in rapid gravity flows of modeling materials at simultaneous identification of the interrelationship coefficient of the granular medium state equation. The granular materials consisting of uniform spherical particles differing dominantly in roughness were used as model materials. It was found out the coefficient is equal to $1 \pm 0.3$ for a wide range of the chute angle and the flow depth of smooth particles. The analogous coefficient value is observed for rough particles when the high chute angle or small bed depth take place only. However, the coefficient value increases more than four times for rough particles at high values of the bed depth and the chute angle close to the angle of repose of the material. This phenomenon is explained hypothetically by the effect of particle rotation.
\end{abstract}

Keywords - granular material; rapid gravity flow; rough chute; state equation; dilatation; granular temperature

\section{Introduction}

Free surface gravity flows of granular media are often encountered in terms of many natural phenomena (debris flows, snow and rock avalanches, sand flows in dunes) and technological operations (loading, unloading and transportation of granular materials in reactors, dryers, granulators, mixers). It is well-known, e.g. [1-3], that granular gravity flows are accompanied by intensive particle interactions, which result in segregation, remixing, migration and other physical effects, influencing essentially on dynamics of technological processes and natural phenomena. As it is

Dolgunin Viktor Nikolaevich

Ivanov Oleg Olegovich

Kudi Andrey Nikolaevich

Tuev Maxim Alekseevich

Tambov State Technical University

Russia clearly shown by Wiederseiner et al. [4], Hill and Fan [3], in order to explain and forecast these effects, the detailed information on micro structural level needs in terms of kinematical and structural flow characteristics. In opposite case, some discrepancies arise, their reasons are not clear.

In the last decades numerous analytical and experimental investigations of such flows were carried out, e.g. Savage [5]; Brennen [6]; Forterre and Pouliquen [7]; Pudasaini [8]; Domnik et al. [9], Windows-Yule et al. [10]. However, as it was truly noted by Jesuthasan and Savage [11], Hill and Fan [3], so far mathematical models of granular flows have not been fully established and, furthermore, the united model of such flows is not available $[7,10]$. Thus, there is a need for reliable experimental data that can be used for verification of the mathematical models.

In order to describe the dynamics of shear flows of particulate solids two basic methods are used. The discrete element methods (DEM) analyze the behavior of individual particles taking into account their interactions. These methods are preferably used when the particle interaction conditions allowing idealization of the factors of internal and external influence on individual particles take place, e.g. for experimental result imitation $[3,10]$. On the contrary, other methods represent the granular medium as a continuum. The continuum approach is widely used to describe the dynamics of flows formed in complex boundary conditions, e.g. geophysical flows of complex geometry $[8,9]$. However, the corresponding models of flow dynamics are based, as a rule, on the assumption of incompressibility and homogeneity of granular media. This assumption leads to depth-averaged model equations which cannot be used for a physically complete description of the flow dynamics without information reduction through the flow depth [3, 9]. Hydrodynamic models based on phenomenological approach and taking into account the microstructural properties of granular media can contribute to the solution of this kind of problem, however, the development of such models is only in the initial stage $[7,10]$.

In the case of rapid granular flow, the phenomenological approach to describe the flow dynamics assumes a wide use of the analogy between the chaotic movement of solid particles and the thermal motion of molecules in the kinetic theory of gases. Many authors have pointed out that rapid shear flows are analogous to molecular gas dynamics, e.g. Savage [5], Ackermann and Shen [12], Hutter et al. [13], Jenkins and Berzi [14], Dolgunin et al. [15]. This allowed some of them to 
apply a well-elaborated kinetic gas theory for solving the dynamics problem of rapid granular flow.

Adapting of a Goodman-Cowin's [16] model of rapid shear flows of granular materials, based on the main statements of the continuous medium mechanics, Savage [17] developed the model for particles rapid gravity flow down an incline. Later, taking into account the evident drawbacks of the continuous medium models Ogawa et al. [18], Jenkins and Savage [19], Ackermann and Shen [12] and Savage [5] developed a model of a rapid gravity flow taking into account some microstructural effects. These authors determined the stress tensor by analyzing momentum translation during particle collision and solving the system of equations on the conservation of momentum and fluctuation energy.

But the assumptions made by the authors led to some errors in the flow description. These errors being particularly apparent in numerical modeling of granular particles flow down a steep incline carried out by Hutter and Sheiwiller [20]. Moreover, they have demonstrated that existing models require an exact statement of boundary conditions, which are difficult to determine.

Ackermann and Shen [12] used geometrical analysis of the shear flow microstructure and came to the conclusion that the gravity flow should be characterized by a considerable lateral mass transfer (quasi-diffusion) which must be taken into consideration for an adequate flow modeling. However, it seems evident that the authors restrict the possibilities of the approach when they proceed from the assumption made that the collision conditions do not depend on the solid phase concentration. The analogous assumption is also used in most analytical investigations of rapid shear flows.

Campbell and Brennen [21] carried out a direct computer modeling of a rapid shear flow of cohesionless nonelastic rough particles. One of the most important results of their computational experiment is the conclusion that the effective coefficient of friction determined as the ratio of shear stress to normal stress depends on the solid phase concentration. Similar results were obtained experimentally by Savage [5].

Savage and Jeffrey [22], Jenkins and Savage [19] used the term of granular temperature to estimate the intensity of chaotic movements and their influence on the flow dynamics. They defined granular temperature as the value being proportional to square of the mean fluctuating velocity component.

Shirko and Sakharov [23] developed an equation describing the particle movement dynamics in rapid shear flows on the basis of the theory of Brownian movement of molecules in liquids. This equation was developed taking into consideration the assumption that the forces acting on the particle are proportional to its velocity and oscillation amplitude. It is obvious that this assumption makes the obtained results restricted for the flows of very high concentration of particulate solids.

Gidaspow [24] showed that there exists an equation of state for particles which establishes the relation between particle pressure, granular temperature and bulk viscosity.
It should be pointed out that in all the above mentioned models the rotary motion of particles has not been considered. The role of rotary movement of particles in kinetic theory of gas-solid flow was defined by Rahaman and Naser [25]. The authors got a negligible effect of particle rotation on different flow properties such as number of collisions, solids pressure etc. It was also found out that the effect of rotation on particulate medium viscosity is quite significant only at low granular temperature.

Dolgunin and Ukolov [26] suggested a granular medium state equation which correlates medium pressure, dilatation and granular temperature. However, analyzing circumstances complicating the use of the kinetic theory of granular material Brennen [6] arrived at the conclusion that the practical kinetic theory of granular materials has been long in development. In this case, the experimental methods investigating local structural and kinematical parameters of rapid granular flows are of great importance. Relevant experimental information will contribute to the development of mathematical models of flow dynamics and serve as a basis for assessing their adequacy.

In the meantime, considerable experimental difficulties complicate the rapid gravity flow study of granular materials. It is rightly pointed out by Jesuthasan and Savage [11] that free-surface granular flows down a steep incline appear to be extremely complicated for an experimental study despite their apparent simplicity. So far there is a need for reliable experimental methods that can be used for the determination of a full complex of local structural and kinematical parameters of such flows. The analysis of the investigation results of such flows shows [5, 20] that the major difficulties arise from the high sensitivity of gravity flows to an internal probe and the largest boundary effects impeding the use of visual investigation methods. At present, these difficulties promote the appearance of new alternative experimental methods based on the use of several penetrating radiations.

Langston et al. [27] have used the tomography measurement by means of the X-ray scanner to observe the nonuniform particles distribution in the moving bed of particulate solids during the hopper discharge. However, the authors note the exceptional complexity of this method to apply it to the medium microstructure research.

In order to investigate the structure of densely packed beds of particulate solids Sederman et al. [28] employed magnetic resonance imaging (MRI). George et al. [29] have carried out three-phase material distribution measurements in the vertical flow using gamma-densitometry tomography and electricalimpedance tomography (ERT). However, all the abovementioned methods using several penetrating radiations are problematic, when the problem of local microstructure determination must be solved.

At present in widespread cases of practice and scientific research the computer tomography measurements are used. Denes et al. [30] carried out computer tomography measurement in a gravity flow of particles during the hopper discharge. The X-ray computer tomograph "Siemens Somation Plus" was used to observe the dynamics of the flow boundary 
change in the course of the discharge process. Unfortunately, in spite of the unique technique this investigation method has not been developed to determine the local microstructure parameters.

Dolgunin and Ukolov [26] suggested an experimental and analytical method of determination of the velocity and concentration profiles in rapid gravity flows of particulate solids on a rough chute. In the present paper this method is used to do research on forecasting properties of the phenomenological equation which correlates medium pressure, dilatation and granular temperature in the course of rapid granular flow on a rough chute. This equation was suggested earlier by Dolgunin and Ukolov [26] and modified by Borschov et al. [31].

\section{Experimental and analytical (unit and method)}

An experimental and analytical method of determination of the velocity and concentration profiles in rapid gravity flows of particulate solids was suggested by Dolgunin and Ukolov [26]. The experimental part of the method consists in sending a granular material down a rough incline and collecting the particles in a tray containing a number of cells (Fig. 1).

In accordance with the method experimental data include the bed depth $h$ at the discharge threshold, time $t$ of the tray filling, the material distribution function $G\left(x_{1}\right)$ along a certain horizontal coordinate axis $x_{1}$ located at a vertical distance $H$ from the discharge threshold and the bed inclination angle $\alpha$ (Fig. 1). Distribution function $G\left(x_{1}\right)$ is determined by means of collecting the particles in a tray containing a number of cells in the course of a steady granular flow on a rough chute [26]. The air drag during particle fall was negligible due to small fall heights $H$ It was tested experimentally by means of the profiles determination for different values of particle fall heights $H$.

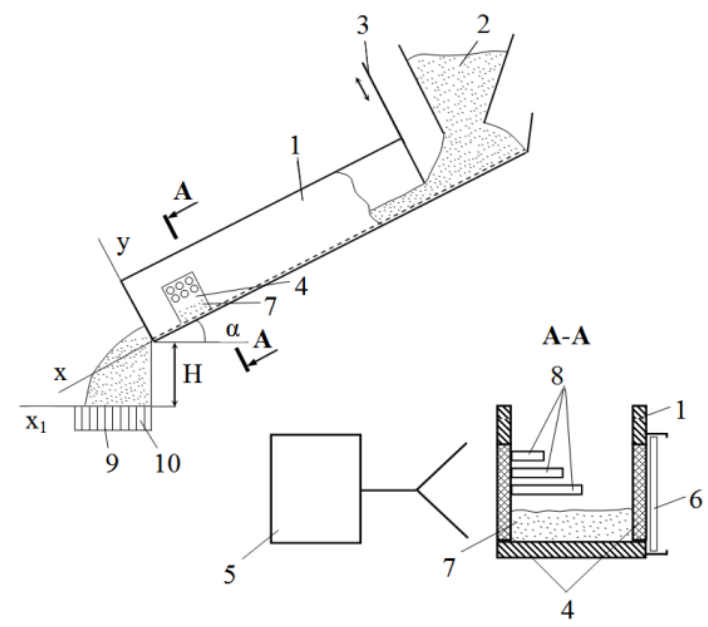

Figure 1. Schematic of the experimental unit:

1 - channel, 2 - dosage device, 3 - gate, 4 - gaps closed by organic glass, 5 $\mathrm{X}$-ray source, 6 - cassette containing the X-ray film, 7 - gravity flow of particles, 8 - control samples of modeling materials having different density, 9 - tray, 10 - cells
The equations connecting the profiles of velocity $u(y)$, fraction of the void volume $\varepsilon(y)$ and the material distribution function $G\left(x_{1}\right)$ in the rapid gravity flows of particulate solids on a rough chute are formulated as follows

$$
\begin{gathered}
|\vec{u}|=\frac{x_{1}-y \sin \alpha}{\cos \alpha \sqrt{\left(H+y \cos \alpha-\left(x_{1}-y \sin \alpha\right) \operatorname{tg} \alpha\right) \frac{2}{g}}} \\
u\left(y\left(x_{1}\right)\right) \rho(1-\varepsilon(y))=G\left(x_{1}\right) .
\end{gathered}
$$

Eqs. (1) and (2) indirectly determine the unknown functions $\varepsilon(y)$ and $u(y)$. Additional data are needed to solve these equations. Because of that, using the hypothesis about an analogy between the parameters of the granular medium under rapid shear and the corresponding parameters of the dense gas the authors have obtained a granular medium state equation.

The equation determines directly the interrelationship between dilatation $\bar{\varepsilon}$, normal pressure $p$ and granular medium temperature $E$ during rapid shear flow of nonelastic cohesionless particles as follows:

$$
p \cdot \bar{\varepsilon}=\chi \cdot E,
$$

where $p(y)=\int_{h-y}^{h} \rho(1-\varepsilon(y)) \cdot g \cdot \cos \alpha d y$ is the hydrostatic pressure analogue; $\bar{\varepsilon}(y)=\frac{\varepsilon(y)-\varepsilon_{o}}{1-\varepsilon(y)}$ is the granular medium dilatation; $\frac{d u}{d y}$ is the shear rate; $\chi^{\prime}$ is the equation coefficient of the granular medium state.

In contrast to the traditional presentation, the granular medium temperature $E$ is defined here as an analogue of the kinetic energy of particles caused by their mutual displacement. Three elementary types of mutual displacements of particles were taking into account (see Borschov and Dolgunin [31]) in order to define the granular medium temperature:

$$
E=E_{s h}+E_{f l}+E_{t r},
$$

where: $E_{s h}$ - the energy caused by the relative velocity of particles to shear direction; $E_{f l}$ - the energy depending on the chaotic fluctuation of particles; $E_{t r}$ - the energy caused by the transversal displacement (mass transfer) of particles.

The granular temperature in the form of eq. (4) reflects the energy effects of the mutual particle motion generated of by gravity shear. The mutual particle motion has a complex form that can be represented as the sum of simple forms of particle motion relative to each other, such as: simple shear, chaotic motion (fluctuation) and transversal mass transfer towards the flow depth direction (see Shen and Ackermann [32]).

The kinetic energy of the relative motion of particles caused by their transversal displacement in two-dimensional shear flow was expressed as follows:

$$
E_{t r}=\frac{1}{4} \rho s V^{\prime} \frac{d u}{d y},
$$

where: $s=\left(b / b_{0}-1\right) d ; b_{0}$ is the geometrical parameter calculated for the least value of $\varepsilon$. 
The specific kinetic energy caused by the particle fluctuation is defined as

$$
E_{f l}=\frac{1}{2} \rho\left(V^{\prime}\right)^{2} .
$$

The kinetic energy of the relative motion of particles in consequence of shear flow is expressed as follows

$$
E_{s h}=\frac{1}{2} \rho(b d)^{2} \cdot\left(\frac{d u}{d y}\right)^{2} .
$$

In Eqs. (5), (6) the fluctuation velocity $V^{\prime}$ is calculated from an equation of energy conservation during particle shear flow on the basis of Ackermann - Shen [32] method. Later, using an original combined hypothesis of particle impact Dolgunin et al. [33] have performed the method evolution avoiding the obvious method drawbacks. This evolution provides calculating the fluctuation velocity as follows

$$
V^{\prime}=\varphi b d \frac{d u}{d y},
$$

where $\varphi$ is the complex depending on the energy dissipation component.

This complex is defined as a function of particle properties, structural and kinematic flow characteristics

$$
\varphi=\left[\frac{(1+\mathrm{k})(0,05+0,08 \mu)(1+\mathrm{s} / \mathrm{d})}{[3 \mathrm{~s} C / 2 d] \rho_{f} / \rho+\mathrm{e}_{\mathrm{c}}}\right]^{1 / 2}
$$

The fraction of kinetic energy dissipated during particle collision $e_{c}$ is calculated in the following way

$$
e_{c}=\left(1-k^{2}\right)+\frac{2}{\pi} \mu(1+k)+0,5 \lambda-0,125 \mu^{2}(1+k)^{2}-\frac{2}{3} \lambda \mu(k+1)-0,125 \lambda^{2}
$$

The coefficient of the tangent velocity reduction at particle collisions $\lambda$ is determined on the basis of the assumption that the impact pulse of the tangent impact consists of the normal and tangential components. This coefficient is defined experimentally and the normal post-impact component of the pulse is determined in accordance with the law of the frontal impact [33].

Thus, the Eqs. (5)-(10) allow to rewrite the granular medium state equation (3) as follows

$$
\bar{p} \bar{\varepsilon}=\chi E=\chi(1 / 2) \rho\left[(b d)^{2}+\frac{1}{2} s \varphi b d+\varphi^{2}(b d)^{2}\right] \cdot\left(\frac{d u}{d y}\right)^{2} .
$$

Eqs. (1), (2) and (11) with due regard to expressions (3) (10) make up a closed system, relative to the functions $u(y)$, $\varepsilon(y), y\left(x_{1}\right)$ and $p(y)$. Thereat $y\left(x_{1}\right)$ is the particle coordinate in the bed, determined by its coordinate after falling on axis $x_{1}$ (Fig.1). The boundary condition for this equation system is formulated as adhesion condition at the rough bottom $y=0$. In this case the boundary condition is formulated in the following way

$$
u(0)=0 ; y(0)=0 .
$$

In order to achieve this "no-slip" condition the chute roughness was equal to half the diameter of the largest particles in the mixture. Then the particle velocity was equal to 0 when these particles contacted with the chute.
The equation system (1), (2) and (11) is solved numerically by successive approximation method [34]. The first approximation step assumes: $\varepsilon(y)=\varepsilon=$ constant.

The adequacy of Eq. (11) was checked by means of comparison of modeling results with experimental data obtained by the X-ray photography method [35]. As it shown in Fig. 2 the standard deviation between experimental and modeling results is equal to $3.8 \%$. This result induced us to estimate the forecasting properties of Eq. (11) in a wide range of rapid gravity flow parameters on a rough chute.

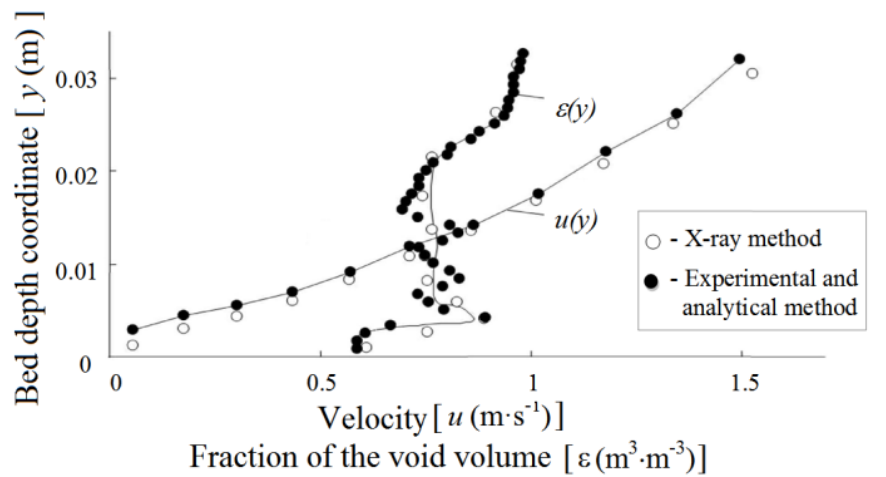

Figure 2. Profiles of velocity $u(y)$ and fraction of the void volume $\varepsilon(y)$ during ceramic balls gravity flow down a rough chute.

The results shown in Fig. 2 allow observing the following two original features of the particle behavior in such flows. The first one consists in the formation of more compact layer areas in the central part of the bed. On the contrary, the peripheral flow areas located at the free surface and the bottom of the bed are characterized by the highest values of fraction of the void volume.

The second feature of the flow is the tendency of particles to organize regular columns especially under low values of the fraction of void volume in the central part of the bed.

The forecasting properties of Eq. (11) were investigated by means of the above-mentioned experimental and analytical method which presupposes the distribution analysis of particles falling from the discharge threshold of the chute (Fig. 1). The profiles of velocity and fraction of void volume have been determined for steady states of rapid gravity flows of modelling materials at simultaneous identification of the interrelationship coefficient $\chi$ of the particulate medium state equation (11).

The uniform cohesionless grain materials consisting of spherical nonelastic particles differing essentially in roughness (glass beads and ceramic balls) were used as modelling media (Table 1). 
Proc. of the Seventh Intl. Conf. on Advances in Civil, Structural and Mechanical Engineering - CSM 2018

Copyright (C) Institute of Research Engineers and Doctors, USA .All rights reserved.

ISBN: 978-1-63248-163-4 DOI: 10.15224/978-1-63248-163-4-14

TABLE I. MODELLING MATERIALS

\begin{tabular}{|c|c|c|c|c|c|c|c|}
\hline \multirow[t]{2}{*}{ Material } & \multirow{2}{*}{$\begin{array}{c}\text { Particle } \\
\text { size, } \\
d \cdot 10^{3} \mathrm{~m}\end{array}$} & \multirow{2}{*}{$\begin{array}{l}\text { Particle } \\
\text { density, } \\
\rho, \mathrm{kg} / \mathrm{m}^{3}\end{array}$} & \multicolumn{3}{|c|}{ Coefficients of } & \multirow{2}{*}{\begin{tabular}{|c|} 
Angle of \\
repose \\
of the \\
material \\
$\alpha_{o}$, \\
deg.
\end{tabular}} & \multirow[b]{2}{*}{$\begin{array}{c}\text { Fraction } \\
\text { of the } \\
\text { void } \\
\text { volume of } \\
\text { dense } \\
\text { packed } \\
\text { particles } \\
\varepsilon_{o}, \\
m^{3} \cdot m^{-3}\end{array}$} \\
\hline & & & $\begin{array}{c}\text { friction } \\
\mu\end{array}$ & $\begin{array}{c}\text { tangent } \\
\text { velocity } \\
\text { loss at } \\
\text { collisions } \\
\lambda\end{array}$ & $\begin{array}{c}\text { restitution } \\
k\end{array}$ & & \\
\hline $\begin{array}{l}\text { Glass } \\
\text { beads }\end{array}$ & 3.385 & 2500 & 0.1 & 0.28 & 0.92 & 26 & 0.37 \\
\hline $\begin{array}{c}\text { Ceramic } \\
\text { balls }\end{array}$ & 6.6 & 2086 & 0.44 & 0.57 & 0.67 & 36 & 0.4 \\
\hline
\end{tabular}

\section{Results and discussion}

An experimental and analytical method, suggested earlier by Dolgunin and Ukolov [26], allowing to obtain the profiles of velocity, fraction of the void volume and test particle distribution along the bed depth during rapid granular flow on a rough chute has been used to investigate the forecasting properties of Eq. (11). The forecasting properties were estimated by means of the coefficient $\chi$ determination as a function of flow parameters. The investigation was carried out for the following range of gravity flow parameters: $\sin \alpha / \sin \alpha_{0}$ $=1.0 \ldots 1.15 ; h / d=4 \ldots 10$, where: $d$ is the particle diameter; $h$ is the bed depth; $\alpha$ is the chute inclination angle, $\alpha_{0}$ is the angle of repose of the material. The investigation results are shown in Figs. 3 and 4 for glass beads and ceramic balls respectively.

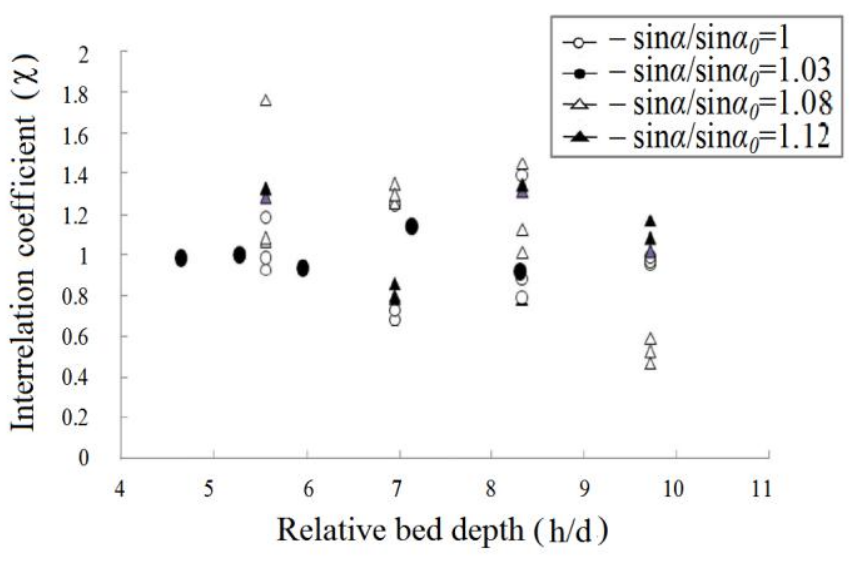

Figure 3. Interrelationship coefficient $\chi$ as a function of the bed depth during rapid gravity flow of glass beads for different values of the chute inclination angle: $\longrightarrow-\sin \alpha / \sin \alpha_{0}=1, \longrightarrow \sin \alpha / \sin \alpha_{0}=1.03$ [31], $\triangle \sin \alpha / \sin \alpha_{0}=1.08$, $\simeq \sin \alpha / \sin \alpha_{0}=1.12$.

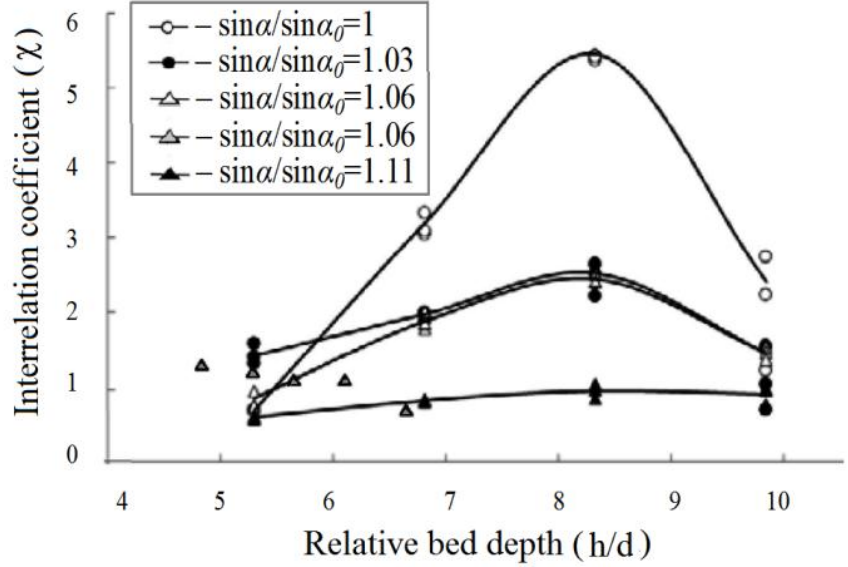

Figure 4. Interrelationship coefficient $\chi$ as a function of the bed depth during rapid gravity flow of ceramic balls for different values of chute inclination angle: $\longrightarrow-\sin \alpha / \sin \alpha_{0}=1, \longrightarrow \sin \alpha / \sin \alpha_{0}=1.03,-\triangle$ $\sin \alpha / \sin \alpha_{0}=1.06, \triangle \sin \alpha / \sin \alpha_{0}=1.06$ [31], $\simeq \sin \alpha / \sin \alpha_{0}=1.1$.

This result allowed us to define the flow conditions when particulate medium state may be described by Eq. (11) at the coefficient $\chi$ value is equal to $1 \pm 0.3$. It was found out that these conditions are observed under the whole range of investigated flow parameters for smooth particles of beads. As to the rough particles (ceramic balls) the analogous conditions are observed only with the high chute inclination angle $(\sin \alpha / \sin \alpha 0>1.1)$ or the small bed depth $(h / d=4 \ldots 6)$. For the rough particles within the range of the bed depth $h / d=7 \ldots 9$ the interrelationship coefficient $\chi$ is greatly influenced by the chute inclination angle. Namely, when the angle decreases from $\sin \alpha / \sin \alpha_{0}=1.1$ to $\sin \alpha / \sin \alpha_{0}=1.0$, the coefficient $\chi$ increases more than four times.

In some way this phenomenon can be explained hypothetically by means of combined analysis of profiles of velocity and fraction of the void volume which have been shown in Fig. 5.

The paradoxical combination of the greatest values of fraction of void volume and the least values of the shear rate at the chute inclination angle $\sin \alpha / \sin \alpha_{0}=1$ allowed us to assume that the observed $\chi$ coefficient increase is explained by the effect of one more elementary form of particle mutual displacements. Probably, this elementary form is the particle rotation which needs to be identified. This supposition is correlated with a conclusion made by Rahaman and Naser [25] that the effect of particle rotation is significant at low shear rates. 


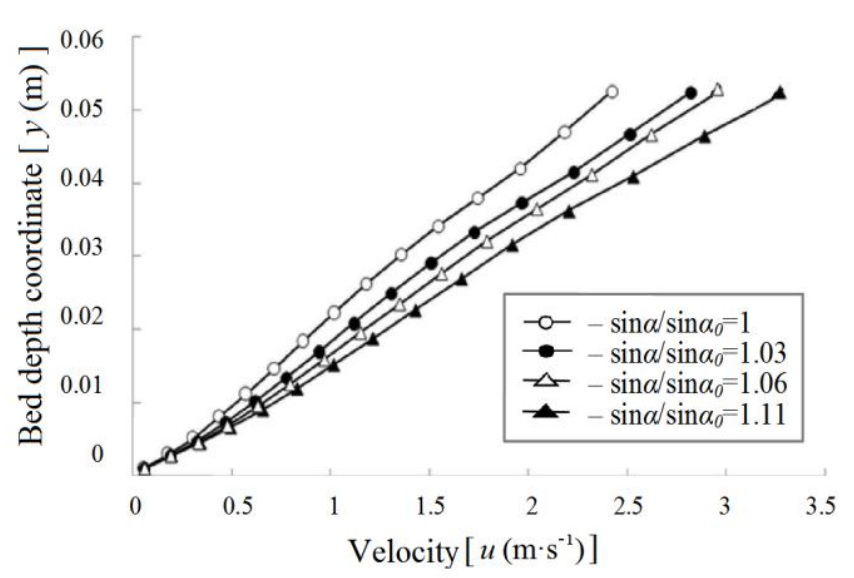

(a)

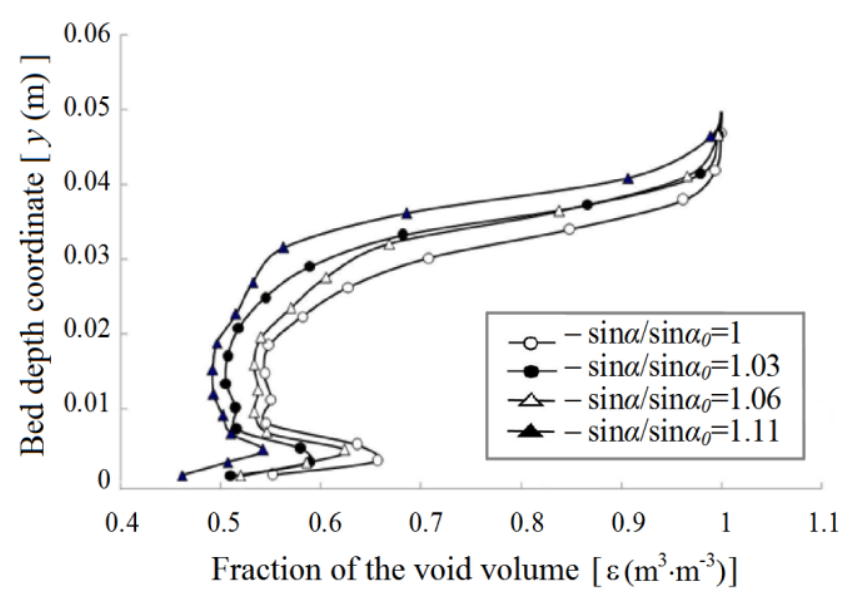

(b)

Figure 5. Profiles of velocity (a) and fraction of the void volume (b) during rapid gravity flow of ceramic balls at the relative bed depth $h / d=8$ for different chute inclination angle $\alpha$.

\section{Conclusions}

On the basis of a formal analogy between the granular medium undergoing rapid shear and a dense gas the interrelationship between the medium dilatation, normal pressure and granular medium temperature in the course of granular flow on a rough chute was investigated.

The granular medium temperature is defined as an analogue of the kinetic energy of particles caused by their mutual displacement in the flow. Three elementary types of mutual displacements of particles were taken into account in order to define the granular medium temperature (kinetic energy), namely:

- energy caused by the relative velocity of particles in shear direction;

- energy depending on the chaotic fluctuation of particles;

- energy caused by the transversal displacement (mass transfer) of particles.
The uniform cohesionless grain materials consisting of spherical nonelastic particles differing essentially in roughness (glass beads and ceramic balls) were used as modelling media.

The investigation was carried out by means of the earlier developed experimental and analytical method. The investigation consisted in the interrelation coefficient $\chi$ determination of the particulate medium state equation as functions of relative values of the bed depth $h / d$ and the chute inclination angle $\sin \alpha / \sin \alpha_{0}$ varied in the values range $4 \ldots 10$ and 1.0...1.15 respectively. It was found out that the coefficient $\chi$ value is equal to $1 \pm 0.3$ into the whole range of investigation flow parameters for smooth particles of beads. As to the rough particles (ceramic balls) the analogous conditions are observed only when the high chute inclination angle $\left(\sin \alpha / \sin \alpha_{0}>1.1\right)$ or the small bed depth $(\mathrm{h} / \mathrm{d}=4 \ldots 6)$ take place. For the rough particles within the range of the bed depth $h / d=7 . . .9$ the interrelationship coefficient $\chi$ greatly depends on the chute inclination angle. Namely, when the angle decreases from $\sin \alpha / \sin \alpha 0=1.1$ to $\sin \alpha / \sin \alpha 0=1.0$ coefficient $\chi$ increases more than four times. This result allowed us to assume that the observed $\chi$ coefficient increase is explained by the effect of one more elementary form of particle mutual displacements. Probably, this elementary form is the particle rotation which needs to be identified.

\section{Nomenclature}

$b=(\pi /(6(1-\varepsilon)))^{0,33}$ - geometrical parameter, $(-)$;

$C$ - drag coefficient for the particle movement in interstitial fluid, (-);

$d$ - diameter of particles, (m);

$E$ - granular temperature, $\left(\mathrm{kg} \cdot \mathrm{m}^{2} \cdot \mathrm{s}^{-2}\right)$;

$g$ - gravity acceleration, $\left(\mathrm{m}^{-2}\right)$;

$H$ - vertical distance between the chute discharge threshold and the tray (Fig. 1), (m);

$h$ - bed depth, (m);

$G\left(x_{1}\right)$ - material distribution function along a horizontal coordinate $x_{1}$ (Fig. 1), $\left(\mathrm{kg}^{-2} \mathrm{~s}^{-1}\right)$;

$k$ - restitution coefficient at collisions, (-);

$P(y)$ - analogue of hydrostatic pressure, $\left(\mathrm{N}^{-2}\right)$;

$s$ - mean distance between particles, (m);

$u(y)$ - flow velocity, $\left(\mathrm{m}^{-1}\right)$;

$\frac{d u}{d y}$ - shear rate, $\left(\mathrm{s}^{-1}\right)$;

$y, x_{1}$ - Cartesian coordinates (Fig. 1);

$\bar{V}^{\prime}$ - fluctuation component of the particle velocity, $\left(\mathrm{m} \cdot \mathrm{s}^{-1}\right)$;

\section{Greek letters}

$\alpha$ - chute inclination angle, (deg.);

$\alpha_{0}$ - the angle of repose of the material, (deg.);

$\varepsilon$ - fraction of the void volume, $\left(\mathrm{m}^{3} \cdot \mathrm{m}^{-3}\right)$;

$\bar{\varepsilon}$ - bed dilatation, $\left(\mathrm{m}^{3} \cdot \mathrm{m}^{-3}\right)$;

$\varepsilon_{0}$ - fraction of the void volume of dense packed particles, $\left(\mathrm{m}^{3} \cdot \mathrm{m}^{-3}\right)$;

$\chi$ - coefficient of the granular medium state equation (11), (-); 
$\lambda$ - coefficient of the tangent velocity reduction at particle collisions, (-);

$\mu$ - friction coefficient, (-);

$\rho$ - particle density, $\left(\mathrm{kg} \cdot \mathrm{m}^{-3}\right)$;

$\rho_{f}$ - density of interstitial medium, $\left(\mathrm{kg} \cdot \mathrm{m}^{-3}\right)$.

\section{References}

[1] J.M.N.T. Gray, M. Shearer, A.R. Thornton, Time-dependent solutions for particle-size segregation in shallow granular avalanches, vol. 462, 2006, pp. 947-972.

[2] V.N. Dolgunin, A.N. Kudy, A.A. Ukolov, Development of the model of segregation of particles undergoing granular flow down on inclined chute, Powder technology, vol. 96, 1998, pp. 211-218.

[3] K.M. Hill, Y. Fan, Granular Temperature and Segregation in Dense Sheared Particulate Mixtures, KONA, vol. 33, 2016, pp. 150-168.

[4] S.N. Widerseiner, N. Andreini, G. Epely-Chauvin, G. Moser, M. Monnereau, J.M.N.T. Gray, C. Ancey, Experimental investigation into segregating granular flows down chutes, Physics of Fluids, vol. 23, 2011, 013301-013310.

[5] S.B. Savage, Granular flows down rough inclines - Review and extension. In Mechanics of granular Materials (ed. J.T. Jenkins and M. Satake), Elsevier Science Publishers, 1983, pp. 261-282.

[6] C.E. Brennen, Fundamentals of Multiphase Flows, Cambridge University Press, 2005, p. 410.

[7] Y. Forterre, and O. Pouliquen,. Flows of Dense Granular Media. Annual Review of Fluid Mechanics, vol. 40, 2008, pp. 1-24.

[8] S.P. Pudasaini, A general two-phase debris flow model. Journal of Geophysical Research: Earth Surface, vol. 117, F3, 2012, pp. 20032012.

[9] B. Domnik, S.P. Pudasaini, R. Katzenbach, S.A. Miller, Coupling of full two-dimensional and depth-averaged models for granular flows. Journal of Non-Newtonian Fluid Mechanics, vol. 201, 2013, pp. 56-68.

[10] C.R.K. Windows-Yule, D.R. Tunuguntla, D.J. Parker, Numerical modeling of granular flows: a reality check, Computational particle mechanics, vol. 3 № 3, 2016, pp. 311-332.

[11] N. Jesuthasan, B.R. Baliga, S.B. Savage, Use of Particle Tracking Velocimetry for Measurements of Granular Flows: Review and Application - Particle Tracking Velocimetry for Granular Flow Measurements, KONA, vol. 24, 2006, pp. 1-9.

[12] N.L. Ackermann, H.H. Shen, Rapid shear flow of densely packed granular materials. In Mechanics of granular Materials (ed. J.T. Jenkins and M. Satake), Elsevier Science Publishers, 1983, pp. 295-304.

[13] K. Hutter, Y. Wang, and S. Pudasaini, The savage-hutter avalanche model. How far can it be pushed? Phil. Trans. R. Soc. A, vol. 363, 2005, pp. 1507-1528.

[14] J.T. Jenkins, D. Berzi, Kinetic theory applied to inclined flows, Granular Matter, vol. 14, №2, 2012, pp 79-84

[15] V.N. Dolgunin, A.N. Kudi, A.A. Ukolov, M.A. Tuev, Rapid granular flows on a vibrated rough chute: behavior patterns and interaction effects of particles, Chemical Engineering Research and Design, vol. 122, 2017, pp. 22-32.

[16] M.A. Goodman, S.C. Cowin, Two problems in the gravity flow of granular materials, J. Fluid Mechanics, vol. 45, 1971, pp. 321-339.

[17] S.B. Savage, Gravity flow of cohesion less granular materials in chutes and channels, J. Fluid Mechanics, vol. 92, 1979, pp. 53-96.

[18] S. Ogawa, A. Umemura, N. Oshima, On the equations of fully fluidized granular materials, J. Appl. Math. Phys, vol. 31, 1980, pp. 482-493.

[19] J.T. Jenkins, S.B. Savage, A Theory for the Rapid Flow of Identical, Smooth, nearly Elastic Particles. J. Fluid Mechanics, vol. 130, 1983, pp. 187-202.

[20] K. Hutter, T. Sheiwiller, Rapid plane flow of granular materials down a chute. In Mechanics of granular Materials (ed. J.T. Jenkins and M.Satake), Elsevier Science Publishers, 1983, pp. 283-293.
[21] C.S. Campbell, C.E. Brennen, Computer simulation of shear flows of granular materials, In Mechanics of granular Materials (ed. J.T. Jenkins and M. Satake), Elsevier Science Publishers, 1983, pp. 313-326.

[22] S.B. Savage, D.J. Jeffrey, The Stress Tensor in a Granular flow at High Shear Rates. J. Fluid Mechanics, vol. 110, 1981, pp. 255-272.

[23] I.V. Shirko, V.A. Sakharov, Phenomenological theory of rapid moving granular media, based on statistical mechanics methods, Theoretical Foundations of Chemical Engineering, vol. 21, 1987, pp. 661-773.

[24] D. Gidaspow, Multiphase flow and fluidization: Continuum and kinetic theory description, Academic press, San Diego, 1994, p. 467

[25] M.F. Rahaman, J. Naser, Kinetic theory of granular flow: effect of rotary movement on solids pressure \& viscosity. In 4-th World Congress on Particle Technology (Full text of paper in CD-Rom). Sydney, Australia, 2002.

[26] V.N. Dolgunin, A.A. Ukolov, Segregation modeling of particle rapid gravity flow. Powder Technology, vol. 83, 1995, pp. 95-103.

[27] P.A. Langston, M.S. Nikitidis, U. Tuzun, D.M. Heyes, Tomographic measurements and distinct element simulations of binary granular flow voidage. In World Congress on particle Technology 3 (Full text of paper in CD). Brighton, UK. 1998.

[28] A.J. Sederman, P. Alexander, L.F. Gladden, Structure of packed beds probed by Magnetic Resonance Imaging, Powder Technology, 117, 2001, pp. 255-269.

[29] D.L. George, K.A. Shollenberger, J.R. Torczynski, Three-phase material distribution measurements in a vertical flow using gamma-densitometry tomography and electrical-impedance tomography, Int. J. Multiphase Flow, vol. 27, 2001, pp. 1903-1930.

[30] B. Denes, J. Szepvolgy, P. Bogner, T. Folder, J. Gyenis, Computer Tomograph Measurements in Shear and Gravity Particle Flows. In 4-th World Congress on Particle Technology (Full text of paper in CD-Rom). Sydney, Australia, 2002.

[31] V.Ya. Borschov, V.N. Dolgunin, P.A Ivanov, Phenomenological Analysis of the Interaction of Nonelastic Incoherent Particles in a Rapid Gravity Flow, Theoretical Foundations of Chemical Engineering, vol. 42(343), 2008.

[32] H.H. Shen, N.L. Ackermann, Constitutive relationships for fluid-solid mixture. Div. Eng. Mech. ASCE, vol. 108, 1982, pp. 748-763.

[33] V.N. Dolgunin, O.O. Ivanov, A.A. Ukolov, Segregation kinetics of particles with different roughness and elasticity under a rapid gravity flow of a granular medium, Theoretical Foundations of Chemical Engineering, vol. 43, 2009, pp. 187-195.

[34] G.I. Marchuk, Methods of numerical mathematics. Applications of mathematics, Springer-Verlag, New York, Heidelberg, Berlin, vol. 2, 1975.

[35] V.N. Dolgunin, V.Ya. Borschov, P.A. Ivanov, Rapid gravity flow of a granular medium, Theoretical Foundations of Chemical Engineering, vol. 39, 2005, pp. 579-585. 